\title{
Application of HTS Current Lead for Superconducting Magnet
}

\author{
Masafumi OGATA \\ Senior Researcher, \\ Ken NAGASHIMA \\ Cryogenic Systems, Maglev Systems Technology Division \\ Masaru IWAMATSU, Ph.D. \\ Senior Researcher, \\ Laboratory Head, Cryogenic Systems, Maglev Systems Technology Division \\ Akihiko INOUE \\ Yamanashi Maglev Test center, Central Japan Railway Company \\ Yoshiki MIYAZAKI \\ Researcher, \\ Cryogenic Systems, Maglev Systems Technology Division
}

\begin{abstract}
The current lead of a superconducting magnet (SCM) is a conductor that connects the superconducting coils to an external power supply. Since the copper alloy generally used for such leads acts as a route for heat leak to the inside of the SCM, the authors developed a high-temperature superconductor (HTS) current lead and evaluated its properties using a measuring system for the HTS current lead. The properties of this lead enable a reduction in length, and the removal of the evaporated helium gas cooling mechanism of the current lead. The advantages of this HTS current lead enable a higher level of SCM performance.
\end{abstract}

Keywords: HTS, bulk, current lead, superconducting magnet

\section{Introduction}

A superconducting magnet (SCM) is a kind of electromagnet supplied with a current, and is electrically connected to an external power supply via a current lead. The SCM features the capacity to generate a strong magnetic field in a system that is more compact than usual electromagnets, but in order to use the superconducting state of materials, it is essential to put the superconducting coil into a sufficiently low-temperature environment. There is thus a need for the current lead of SCM to conduct current reliably as well as to minimize the amount of heat that leaks from it.

\section{Purpose of this study}

Copper alloy is generally used as the material for the SCM's current lead due to its high conductivity and ready availability. However, although it is a good electrical conductor, it also conducts heat relatively well, and Joule heat in a copper alloy of limited resistance cannot be disregarded in high-current transport.

The authors therefore turned to high-temperature superconducting bulk (HTS bulk) which is independent of metallic materials. HTS bulk is an ideal electrical conductor and reaches a superconducting state in the lowtemperature environment of the SCM. In addition, its ceramic constitution makes it considerably less thermally conductive than metallic material. HTS bulk therefore represents a promising material with satisfactory char- acteristics in terms of the electric and thermal performance required for the SCM current lead.

In view of the above, the authors set the purpose of this study to achieve a higher overall SCM performance by introducing a current lead for the SCM applying the HTS bulk.

\section{Current lead composition of this study}

\subsection{Comparison with the conventional composition}

Figure1 shows the composition of the current lead for the SCM. There is a thermal anchor between the HTS lead on the low-temperature side and an unsteady type lead on the high-temperature side. It is possible with this setup to reduce the lead length and to remove the gascooling tube of the conventional system.

\subsection{HTS lead}

The HTS lead made of HTS bulk is installed between a thermal anchor $(77 \mathrm{~K})$ and a superconducting coil (4.2 $\mathrm{K})$. This temperature condition makes it possible to maintain the superconducting state of the HTS bulk.

\subsection{Unsteady type lead}

An unsteady type lead is installed between a thermal anchor and a terminal of normal temperature. Since this is a temperature domain that cannot maintain a super- 


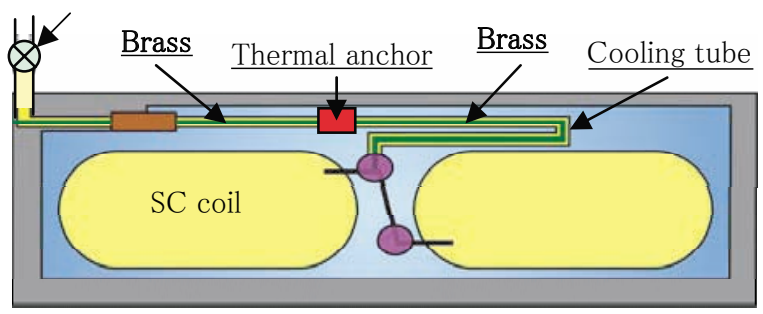

(a) Previous

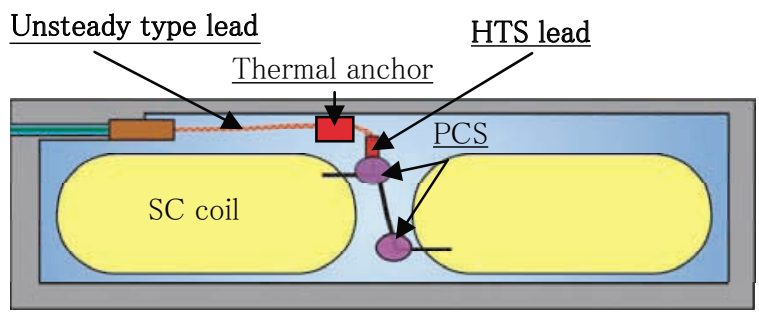

(b) Present setup

Fig. 1 Schematic comparison of current lead composition

conducting state with current HTS bulk performance, a conventional copper alloy lead is used in this section. The removal of the gas-cooling tube also requires a deliberate design for this section lead to minimize the heat load of the total Joule heat and conduction heat in current transportation.

Since the SCM for the superconducting MAGLEV has a built-in permanent current switch (PCS) premised on permanent current mode operation, steady transportation of current to the lead is unnecessary. For the current lead of these SCM, the heat load design during unsteady transportation of current at the time of magnetization or demagnetization is an important issue which is why the lead is called as an unsteady type lead. Conversely, a lead with an optimal heat load design for steady transportation of current is called as a steady type lead.

\section{Development of HTS lead}

\subsection{HTS lead composition}

Since the current lead for SCM is a part of a device that generates a strong magnetic field, the property of stable superconductivity under intense magnetic field is required for HTS bulk. Rare-earth (RE) HTS bulk (RE$\mathrm{Ba}-\mathrm{Cu}-\mathrm{O}$ ) featuring a high critical current density in strong magnetic fields was therefore adapted for the HTS bulk material, and Dysprosium (Dy) was adopted as the rareearth element. Figure2 shows an exterior view of the HTS lead, which has an exterior size of $130 \mathrm{~mm}$, a width of 20 $\mathrm{mm}$, and a thickness of $10 \mathrm{~mm}$. Its constitution allows the direction of current transport in the HTS lead and the direction of the ab-plane of the crystal structure in the HTS bulk material to become the same. The entire HTS lead is formed by vacuum impregnation of epoxy resin, thus increasing the mechanical properties of the HTS bulk ${ }^{1}$.

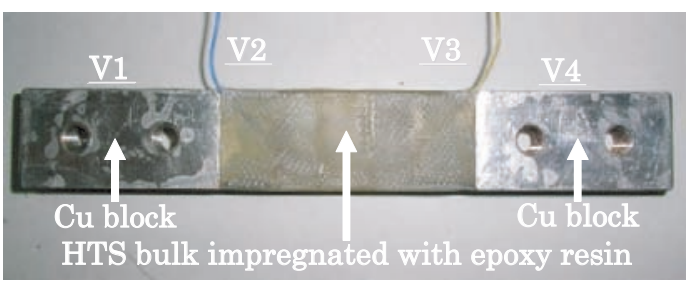

Fig. 2 HTS current lead

\subsection{Properties measurement system for HTS lead}

Figure3 delineates a schematic diagram of this system, and Table 1 describes the principal specifications. Using a permanent magnet (Nd-Fe-B) where a magnetic field of $0.5 \mathrm{~T}$ is impressed to a space $50 \mathrm{~mm}$ wide, $36 \mathrm{~mm}$ high and $90 \mathrm{~mm}$ long, a maximum transport current of $1,000 \mathrm{~A}$ is achieved. The direction of the magnetic field impressed can be set up in arbitrary directions perpendicular to the direction of current transport by rotating the HTS lead for evaluation. Using two GM cryocoolers and heaters for temperature control, it is possible to set the temperature of both ends of the HTS lead individually, from $40 \mathrm{~K}$ to $100 \mathrm{~K}$ for the hot end and from $9 \mathrm{~K}$ to 40 $\mathrm{K}$ for the cold end of the HTS lead. The heat invasion of

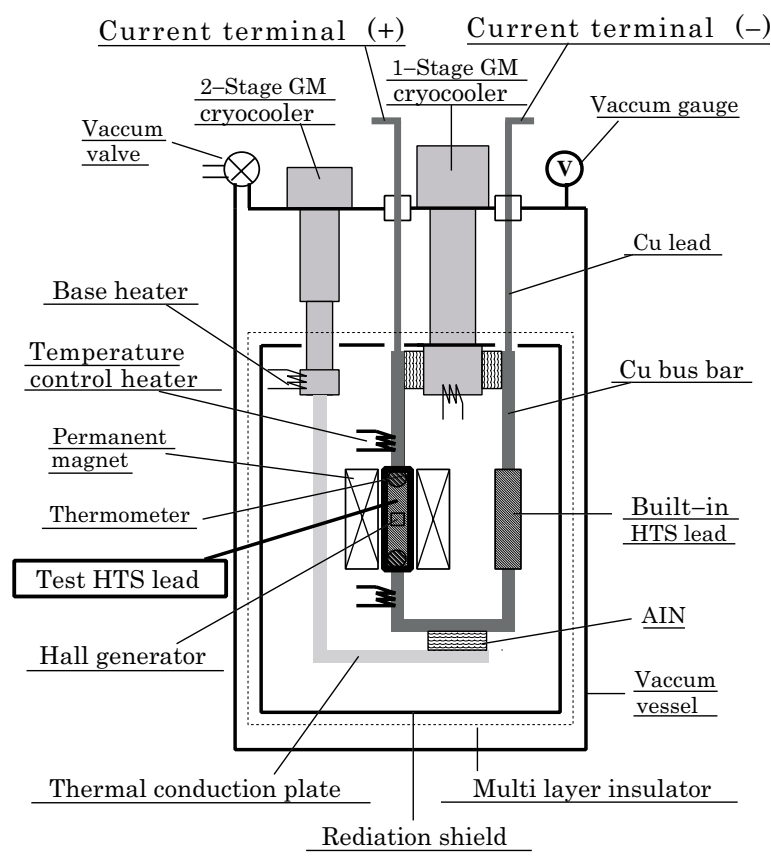

Fig. 3 Schematic diagram of the properties measurement system for the HTS lead

Table 1 Specifications of the properties measurement system for the HTS lead

\begin{tabular}{|l|l|}
\hline Transport current & $1,000 \mathrm{~A}(\max )$ \\
\hline Temperature & $\begin{array}{l}40-100 \mathrm{~K}(\text { Hot end of HTS lead }) \\
9-40 \mathrm{~K}(\text { Cold end of HTS lead })\end{array}$ \\
\hline Magnetic field & $0.55 \mathrm{~T}(\max )$ \\
\hline Measurement of heat leak & $1 \mathrm{~W}(\max )$ \\
\hline Test space for HTS lead & $\begin{array}{l}50 \times 50 \times 300 \mathrm{~mm} \\
(50 \times 36 \times 90 \mathrm{~mm}: 0.5 \mathrm{~T} \text { warranty })\end{array}$ \\
\hline
\end{tabular}


radiation is controlled by arranging a multilayer insulator and a radiation shield plate. It is also possible to measure heat leak in the HTS lead by modifying the busbar composition. The time taken for a sample to reach the minimum attainment temperature is then approximately 24 hours after starting the cryocooler.

\subsection{Test result}

\subsubsection{Critical current measurement (with different temperatures at each end of the lead)}

Figure4 shows the situation of critical current measurement using a commercial HTS ${ }^{2)}$ lead. The hot end of the lead was set at $88 \mathrm{~K}$ and the cold end at $20 \mathrm{~K}$, and a magnetic field of $0.55 \mathrm{~T}$ was impressed in the c-axis direction of the HTS bulk material. A voltage of 5 microvolts, equivalent to the normal phase transition criterion of $1 \mathrm{microvolt} / \mathrm{cm}$, was generated at $954 \mathrm{~A}$. The temperatures of each end of the HTS lead were thus arbitrarily set, and an understanding of the critical properties of the HTS lead was enabled by measuring the voltage of the normal phase transition.

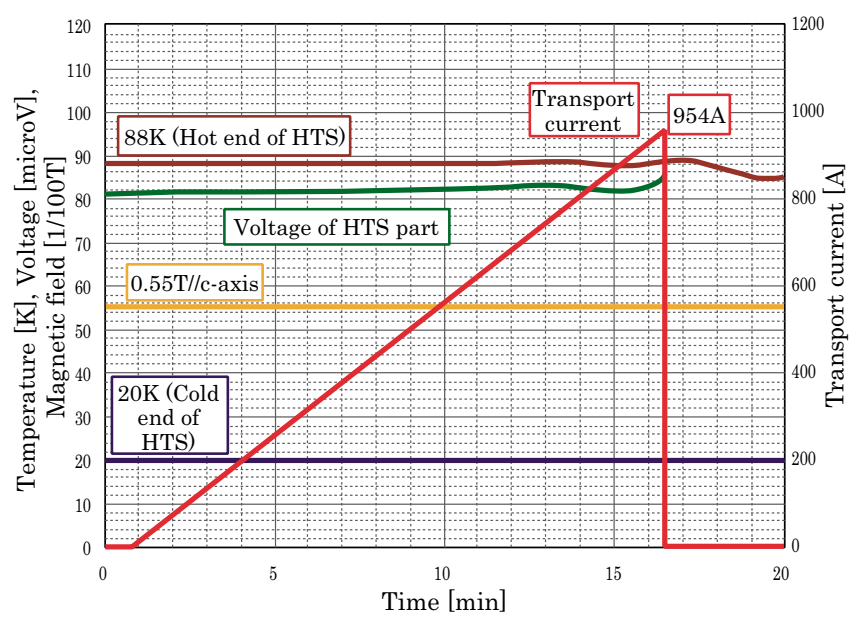

Fig. 4 Test result of critical current measurement with different temperatures at each end of the HTS lead

\subsubsection{Critical current measurement (with the same temperature at each end of the lead)}

Critical current measurement was carried out using the HTS lead as shown in Fig. 2 with identical temperatures at both ends, with the magnetic field impressed in the direction vertical to the c-axis. The I-V characteristics at $85 \mathrm{~K}$ of both ends are shown in Fig.5, with an impressed magnetic field of $0.5 \mathrm{~T}$ and a current sweep rate of $20 \mathrm{~A} / \mathrm{s}$. Here, we decided to transport the current from the left to the right of Fig.2, and to call the potential of the plus-side copper electrode V1, the potential of the plusside HTS bulk V2, the potential of the minus-side HTS bulk V3, and the potential of the minus-side copper electrode V4. The voltage began to rise as it approached 600 $\mathrm{A}$, and started to rise rapidly thereafter. This rapid rise in the voltage shows the transition to a normal conducting state from the superconducting state of the HTS bulk.

The results of critical current measurement in which

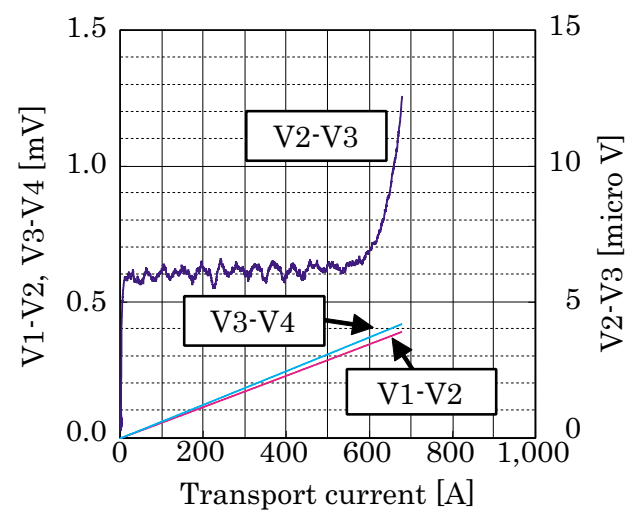

Fig. 5 Test results of I-V characteristics at $85 \mathrm{~K}$ for both ends of the HTS lead (Fig.2) with an impressed magnetic field of $0.5 \mathrm{~T}$ perpendicular to the $\mathrm{c}$-axis

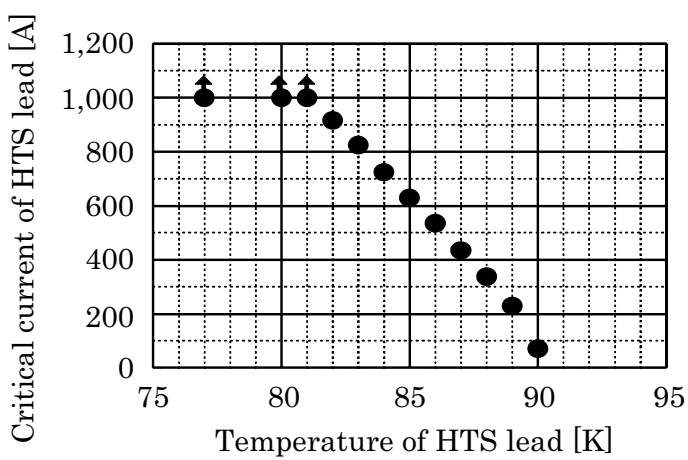

Fig. 6 Test results of critical current in the HTS lead (Fig.2) with an impressed magnetic field of $0.5 \mathrm{~T}$ perpendicular to the c-axis

temperature conditions were changed from $77 \mathrm{~K}$ to $90 \mathrm{~K}$ are shown in Fig.6. The properties of a critical current decreases with a temperature rise in a domain of more than $81 \mathrm{~K}$ were acquired for the first time with a HTS lead.

\section{Development of unsteady type lead}

\subsection{Thermal analytical model}

Analytic study was carried out into the ratio between the cross section and length of the unsteady type lead to minimize the combined heat load from Joule heat and conduction heat when the current is transported.

Table 2 indicates the transport current pattern used for the analysis, while Fig.7 delineates the analytical model. The material for the unsteady type lead is brass, and the temperature of the hot end (TH) of the lead is $300 \mathrm{~K}$, with the cold end (TL) at $80 \mathrm{~K}$.

The basic composition of the unsteady type lead is set with cross section $A=60 \mathrm{~mm}^{2}$ and length $L=72 \mathrm{~mm}$, and for the sake of simplicity $A$ and $L$ at this point are set to $A=1.00$ and $L=1.00$. 
Table 2 Transport current pattern for simulation

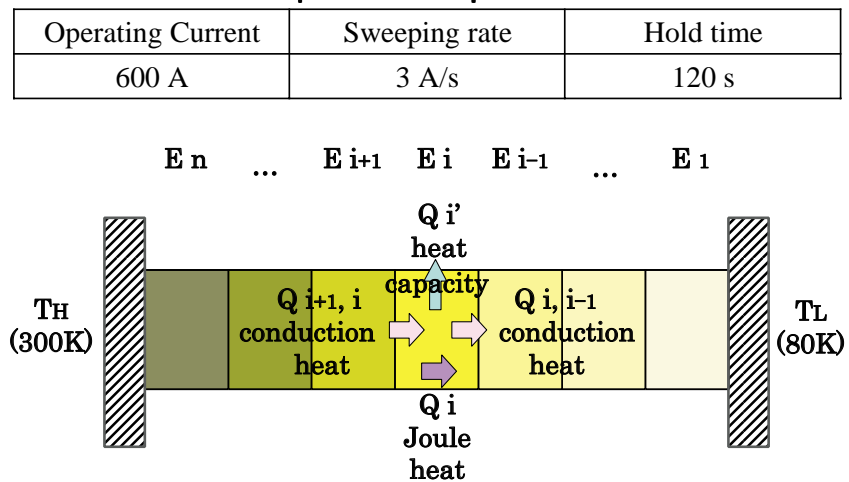

Fig. 7 Analytical model

\subsection{Simulation results}

Figure 8 shows the results under the condition of $A<=1$. As is apparent from the figure, the maximum heat load (Qmax) turns a minimum value of $27 \mathrm{~W}$ at $L=1$. Since the theoretical optimum heat load (Qopt) of the optimized steady type lead $(I L / A=720 \mathrm{~A} / \mathrm{mm}$, where $I$ is the maximum transport current) is $27 \mathrm{~W}$, it turns out that for $A<=1$ the heat load cannot be less than Qopt for every $L$.

Conversely, Fig.9 shows the results under the condition of $A>1$. Qmax cannot be less than Qopt when $L<1$, but where $L>=1$ and $A>=1.5$, Qmax begins to decrease. In the conductor design of the unsteady type lead below Qopt, it therefore turns out that values of $A>1.5$ and $L>1$ are necessary. In addition, it also turns out that the combination of $A$ and $L$ is selectable at the target of Qmax. As

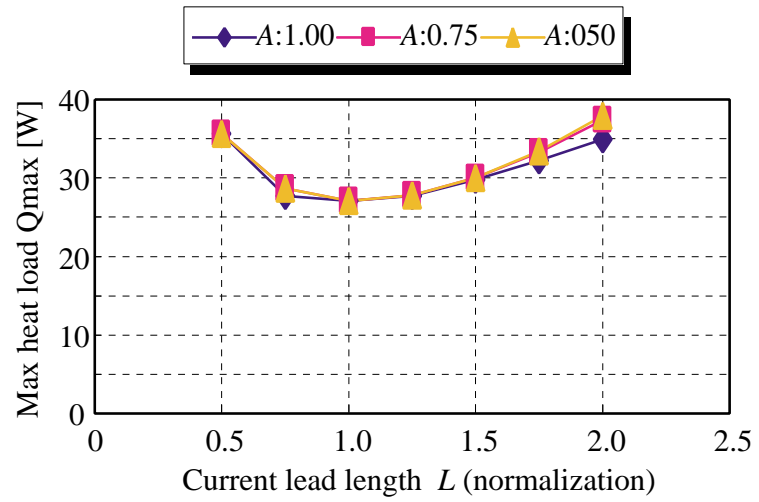

Fig. 8 Simulation results $(A<=1)$

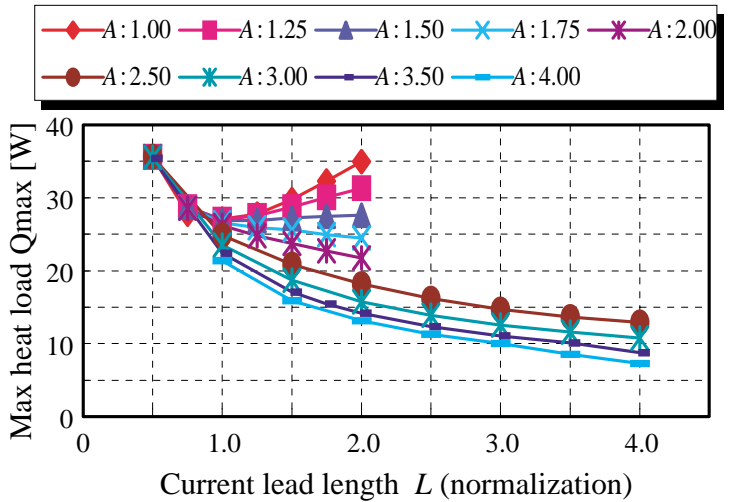

Fig. 9 Simulation results $(A>1)$

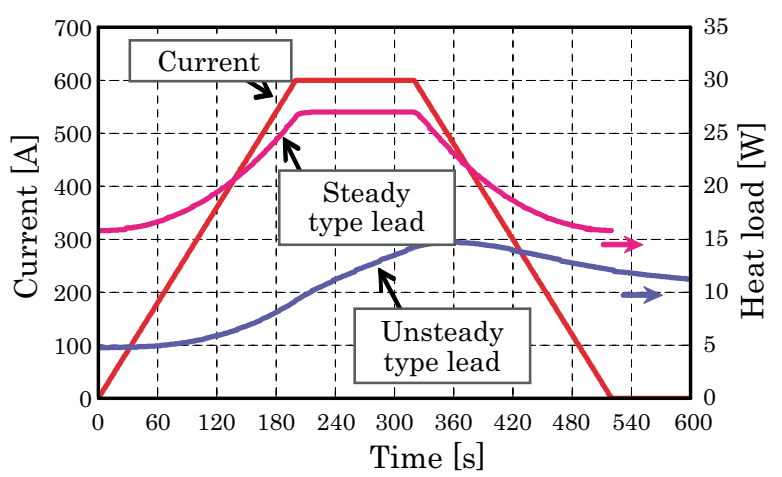

Fig. 10 Heat load simulation results of the unsteady type lead where $A=2.5$ and $L=3.0$

an example, Fig.10 shows the results of heat load simulation of the unsteady type lead where $A=2.5$ and $L=3.0$. The heat load turns $15 \mathrm{~W}$, which is less than the $27 \mathrm{~W}$ value for the steady type lead.

\section{Development of magnetic field generator with HTS lead}

As the initial stage of SCM development with HTS lead, we developed a magnetic field generator, which is part of the electromagnetic vibrating apparatus for the ground coil of superconducting MAGLEV ${ }^{3)}$. The HTS lead is included in this generator without the unsteady type lead. Figure11 shows an exterior photograph of the generator, and Table 3 presents primary specifications.

Although the HTS lead and the copper alloy leads were structured to enable cooling by evaporation gas of

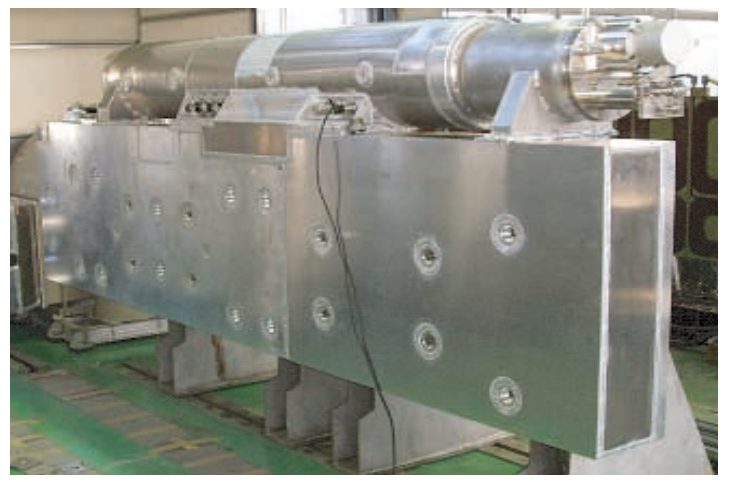

Fig. 11 Magnetic field generator with HTS lead

Table 3 Specifications of Magnetic field generator with HTS lead

\begin{tabular}{|c|c|}
\hline Magnetomotive force & $800 \mathrm{kA}$ \\
\hline External dimensions & $3,430 \times 1,235 \times 490 \mathrm{~mm}$ \\
\hline Coil conductor & $\mathrm{NbTi}$ \\
\hline Coil dimensions & $1,070 \times 500 \mathrm{~mm}$ (racetrack shape) \\
\hline Current lead & HTS bulk $(4 \mathrm{~K} \sim 80 \mathrm{~K})$ \\
\cline { 2 - 2 } & Brass $(80 \mathrm{~K} \sim 300 \mathrm{~K})$ \\
\hline HTS lead dimensions & $90 \times 40 \times 10 \mathrm{~mm}$ \\
\hline Cryocooler & GM+JT $(4 \mathrm{~K} \mathrm{ref.}), \mathrm{GM}(80 \mathrm{~K}$ ref. $)$ \\
\hline
\end{tabular}


liquid helium in the generator, the current transport test was carried out without gas cooling. It was verified that no problems were observed in the current transport properties of the HTS lead without gas cooling.

\section{Future plans}

The authors plan to improve the performance of the HTS lead by accumulating sufficient performance data using the properties measurement system for the lead, and utilizing the results towards the development of a superior design. We also plan to advance development of the unsteady type lead according to conductor design guidelines obtained through thermal analysis to verify the required performance. Then, a SCM current lead that combines both these types of lead will be developed and applied to existing SCM.

In addition, for practical application of the HTS lead for SCM, we will also develop a method of evaluating the reliability and durability of the lead.

\section{Summary}

The authors studied on the SCM for the further performances of the constitution and evaluation method of the current lead for the SCM.

An HTS lead made from the rare-earth HTS bulk was developed.

A properties measurement system for the HTS lead was developed, and the critical current properties of the lead under continuous temperature conditions were acquired for the first time using this system.

Through heat load simulation, a guideline of the de- sign for the unsteady type lead conductor was analytically acquired.

A magnetic field generator with an HTS lead was developed.

We plan to apply a current lead consisting of the HTS lead and the unsteady lead to the SCM. In addition, for practical application, we will also develop a method of evaluating the reliability and durability of the HTS lead.

\section{Acknowledgement}

The authors would like to express their gratitude to the staff of the Toshiba Corporation who manufactured the properties measurement system and the magnetic field generator.

This work is financially supported by the Ministry of Land Infrastructure and Transport.

\section{References}

1) Herai, T. Tomita, M. and Nagashima, K., "Development of Resin-Impregnated HTS Current Leads for MAGLEV," Journal of the Cryogenic Society of Japan, Vol.39, No.3, pp.80-84, 2004.

2) Nippon Steel Corporation Advanced Technology Research Laboratories, "1,000 A class at $77 \mathrm{~K}$ (1-K type) current lead," QMG Current Lead Technical Data, pp.2, 2005.

3) Yanase, Y. Sasaki, K. Yamashita, T. Miyazaki, Y. Nagashima, K. Ogata, M. Iwamatsu, M. and Inoue, A., "Development of Magnetic Field Generator with HTS Current Lead (3)," abstracts of Cryogenic Society of Japan Conference, Vol.73, pp.116, 2005. 